\title{
Use of Short Sensory Profile to Study the Sensory Pattern of Performance across Various Disability Groups
}

\author{
Anita Gupta \\ All India Institute of Physical Medicine and Rehabilitation, Mumbai, Maharashtra, India
}

\begin{abstract}
Use of Short Sensory Profile to study the sensory pattern of performance across various disability groups Objectives - The purpose of this study was to detect whether the factors or items in short sensory profile can differentiate:1) Children with Pervasive Developmental Disorders from children with Cerebral Palsy and sensory issues. 2) C.P. children with sensory issues and without sensory issues. 3) C.P. children with no sensory issues with typically developing children in expressing their sensory responses. Method : 100 children in following categories were assessed on short sensory profile: Group 1 - 22 Typically Developing children, Group 2 - 21 Cerebral Palsy children with no sensory issues, Group 3 - 40 Cerebral Palsy children with sensory issues, Group 4 - 17 children with Pervasive Developmental Disorders. The caretakers responded to the questions asked by the therapist in the questionnaire of short sensory profile. Results and Conclusion: Short sensory profile can discriminate children with sensory issues from the other groups. Responses of Cerebral Palsy children with sensory issues are different from Cerebral Palsy children without sensory issues. Typically developed children are comparable to Cerebral Palsy children without sensory issues on short sensory profile.
\end{abstract}

Keywords: Cerebral Palsy, Sensory Issues, Sensory Profile

\section{Introduction}

In paediatric practise areas, it is often found that children with cerebral palsy of any subtype, with any topographical involvement, have varied neurobehavioral responses. From a sensory integrative perspective, an underlying facet of many of the behaviours observed in children with disabilities is to either generate or avoid sensory stimulation.

Over responsivity is when someone becomes agitated if touched i.e. tactile defensiveness or lack of responsivity is said to be there when one must be tapped on the shoulder several times to gain attention.

Children with Cerebral Palsy, during their therapy sessions and at other times, exhibit maladaptive behaviour, like constant crying with no obvious reason, poor sleeping pattern, and lack of co-operation during therapy, poor tolerance to orthotic appliances or tendency to walk on toes despite no musculoskeletal compromise etc.

In typical SI development, movement and learning depend on the ability to take sensory information from the environment, process it in the Central Nervous System, and use the environmental information to plan and organize behaviour.

Unfortunately, these CP children may experience stress in the course of everyday occupations because processes that should be automatic or accurate are not. It may be stressful, for example, to simply maintain balance when sitting in a chair. The child is aware of these difficulties and becomes frustrated by frequent failure when confronted with ordinary tasks that come easily for other children.

Thus, this study was primarily conceptualised in a top down approach to investigate the sensory performance behaviour of Cerebral Palsy children.

\section{Objectives of the Study}

a) To detect the difference on Short Sensory profile so as to discriminate typically developing children (referred to as TD henceforth) and Cerebral Palsy children without sensory issues (referred to as CPNO).

b) TD children and Cerebral Palsy children with sensory issues (referred as CPSI henceforth).

c) TD children and children with Pervasive Developmental Disorders (referred as PDD).

d) Between CPSI and CPNO and between

e) CPSI and PDD.

\section{Review of Literature}

- Sensory processing behaviours of children ages 3-6 years with and without autism gathered via parent report on the Sensory Profile have been compared by S. Tomcheck and Winnie Dunn (2007). Sensory profile of children with autism was significantly different from the sample without autism on eight of ten factors viz. sensory seeking, emotionally reactive, low endurance/tone, oral sensitivity, inattention/distractibility, poor registration, fine motor /perceptual skills and others. The greatest difference reported on the under responsive, seeks attention, auditory filtering and tactile sensitivity sections. Behavioural features of children with autism attributed to sensory processing qualitatively from children who are typically developing or those with other developmental disorders.

- Adrian and colleagues used observations and frequency counts of behaviours during a structured play session to differentiate between children who are typically developing, children with Mental Retardation and children with autism and very low developmental ages. Although behaviours overlapped between the groups, nine behaviours discriminated children with autism from both typically developing and children with mental retardation. These were - rubbing surfaces, finger flicking, body 


\section{International Journal of Science and Research (IJSR) \\ ISSN (Online): 2319-7064 \\ Index Copernicus Value (2013): 6.14 | Impact Factor (2015): 6.391}

rocking, repetitive jumping, decreased eye contact, limited or inappropriate social smile and laugh, using objects ritualistically, ignoring objects and absent response to stimuli.

- These findings were replicated by Rapin (1996) who found that atypical sensory modulation and motor stereotypes discriminated children with autism from children with other developmental disability. A recent study (Rogers, Hepburn and Wehner (2003)), assessed parent report of sensory reactivity of 102 young children across four groups : autism (26), fragile X syndrome (20), developmental disabilities (32), typically developing children (24). Findings indicated that the groups of autism and FXS had significantly more responses overall than the other 2 groups. Also, this abnormal sensory reactivity had a significant relationship with overall adaptive behaviour.

- Jerome and A. Vaishampayan in their study of sensory processing abilities of children with $\mathrm{CP}$ and typical children on the sensory profile found that 40 of 125 items discriminate and 7 of 14 components on the sensory profile showed significant difference between children with CP and typical children. Analysis of each item on the sensory profile suggested that frequency of responses in children with CP showed lower mean value in 88 items, indicating that children with $\mathrm{CP}$ have more sensory processing difficulty when compared with typical children.

- In the study by R.Wathing, J. Deitz and O. White on comparison of sensory profile, scores of young children with and without autism spectrum disorders, it was found that the performance of children with autism was significantly different from that of children without autism on 8 of 10 factors.

- S.Pedankatti (2005) has compared the performance of children with and without learning disability on the sensory profile tool. The 99 items (Dunn 1994) sensory profile tool discriminates between children with and without learning disability. $65 \%$ of the 99 items were to be uncommon behaviours in children without learning disability.

\section{Methodology}

The primary variable in this study was reported behavioural sensory processing measured by the Short Sensory Profile (SSP).

Data Collection was done by taking children with Cerebral Palsy for the study. Their age ranged from 3 years to 12 years. Children with Pervasive Developmental Disorders were autistic spectrum disorders, fragile $\mathrm{X}$ syndrome and other related disorders present in this group.

Short sensory profile was the tool used for this study. The SSP is a 38 item caregiver report. There are seven sections on the SSP. They are - Tactile sensitivity, Taste/smell sensitivity, Movement sensitivity, Under-responsive/seeks sensation, Auditory Filtering, Low energy/Weak, Visual/Auditory sensitivity. 38 questions are framed under these seven sections.

\section{Scoring system:}

Each response is scored according to the following chart: Always = 1 point (When presented with the opportunity your child always responds in this manner, $100 \%$ of the times).Then there is frequently( 2 points $=80 \%)$, occasionally( 3 points $=60 \%)$ and seldom( 4 points $=20 \%)$ and in the last there is Never $=\mathbf{5}$ points (When presented with the opportunity your child always responds in this manner, $0 \%$ of the times).Frequent behaviours receive lower scores, therefore children get lower scores for undesirable performances and higher scores for desirable performances.

\section{Test Procedure}

The caretakers of children ranging between $3-12$ years were identified for the study and were given the Short Sensory Profile to fill in. The questionnaire and five point scoring was explained by the therapist. Questions were translated in the local language if the caretakers found difficulty to understand the English language used for the questions. Completed forms were collected and taken for statistical analysis.

\section{Data Analysis and Results}

From table 1 it is seen that there were 100 children included in the study.

Table 1

\begin{tabular}{|l|r|c|r|r|r|}
\hline & TD & CPNO & CP5I & PDD & Total \\
Males & 14 & 11 & 27 & 11 & 63 \\
\hline Females & 8 & 10 & 13 & 6 & 37 \\
\hline Total & 22 & 21 & 40 & 17 & 100 \\
\hline
\end{tabular}

There were maximum children in the CPSI group (40), followed by children with TD group(22). The children with CPNO were next in order with 21 children included in the group and PDD group had the least number of subjects totalling to 17 in the group. Males outnumbered the females in all the four groups.

Next, as done by the authors of SSP, 3 categories were made. They were typical performance group, Probable difference group and definite difference group. The score of each child was calculated section wise and the percentage of children in each category (typical, CPNO, CPSI, PDD) from each group(typical, probable, definite) was found.. Performance on the SSP for all the four groups is summarized in Table 2. 
International Journal of Science and Research (IJSR)

ISSN (Online): 2319-7064

Index Copernicus Value (2013): 6.14 | Impact Factor (2015): 6.391

\begin{tabular}{|c|c|c|c|c|c|c|c|c|c|c|c|c|}
\hline & \multicolumn{4}{|c|}{ TYPICAL PERFORMANCE } & \multicolumn{4}{|c|}{ PROBABLE DIFFERENCE } & \multicolumn{4}{|c|}{ DEFINITE DIFFERENCE } \\
\hline & TYPICAL & CPNO & CPSI & PDD & TYPICAL & CPNO & CPSI & PDD & TYPICAL & CPNO & CPSI & PDD \\
\hline Tactilesensitivity & 72.72 & 62 & 42.5 & 29.41 & 18.18 & 8.52 & 12.5 & 11.76 & 9 & 28.57 & 45 & 58.82 \\
\hline $\begin{array}{l}\text { Taste/Smell } \\
\text { sensitivity }\end{array}$ & 81.81 & 80.9 & 42.5 & 58.82 & 13.6 & 4.76 & 2.5 & 11.76 & 4.5 & 14.28 & 7.5 & 29.41 \\
\hline $\begin{array}{l}\text { Movement } \\
\text { sensitivity }\end{array}$ & 80.36 & 47.61 & 35 & 29.41 & 4.5 & 23.80 & 10 & 11.76 & 9 & 28.52 & 55 & 58.82 \\
\hline $\begin{array}{l}\text { Underresponsive/s } \\
\text { eeks sensation }\end{array}$ & 68.18 & 33.33 & 32.5 & 17.64 & 9 & 4.76 & 10 & 5.88 & 22.72 & 42.85 & 57.5 & 76.47 \\
\hline Auditory Filtering & 81 & 76.19 & 40 & 17.64 & 13.6 & 14.28 & 25 & 35.29 & 4.5 & 9.52 & 35 & 47.05 \\
\hline Low energy/Weak & 81 & 38.09 & 22.5 & 47.05 & 4.5 & 9.52 & 5 & 5.88 & 13.6 & 47.61 & 67.5 & 41.17 \\
\hline Visual /Auditory & 90 & 85.7 & 52.5 & 58.82 & 9 & 9.52 & 37.5 & 29.41 & 0 & 4.76 & 17.5 & 11.76 \\
\hline Total & 77.27 & 38.09 & 12.5 & 11.76 & 13.6 & 23.80 & 20 & 17.64 & 9 & 38.09 & 67.5 & 70.58 \\
\hline
\end{tabular}

On the SSP, a definite difference indicates scores greater than 2 standard deviations from the mean for children who were typically developing (in the questionnaire form)A probable difference indicates scores greater than 1 but less than 2 standard deviations from the mean.

The total number of CPSI children in definite difference group was $67.5 \%$,closer to the PDD group which was $70.5 \%$. This is in contrast to the CPNO and typically developing children, who were only $38 \%$ and $9 \%$ respectively, in the definite difference group.

For low energy/weak and visual/auditory processing sections in the definite difference group the CPSI group exceeded in percentage to the PDD group. In contrast to this, the CPNO group had only $4.7 \%$ representation in definite difference category for visual /auditory processing. However, for low energy/ weak, the representation of CPNO children in definite difference category was $47.61 \%$, which was more than the PDD group. This is explained by the existence of motor disability in them and not seen in PDD group.

The percentage of CPSI was least in the typical performance group for low energy/weak, taste/smell sensitivity and visual auditory processing closely followed by the PDD group, whereas the CPNO group was better than both these groups. The percentage of children in this category was closer to typically developing children in taste /smell sensitivity, visual/auditory processing and auditory filtering.

The representation of CPSI and CPNO group in typical performance group for under responsive / seeking sensation section is comparable.

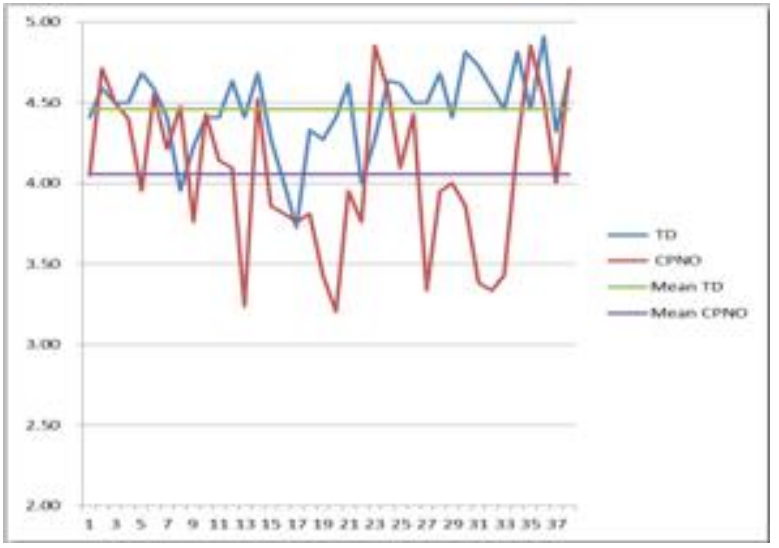

Graph 1

Graph 1shows the comparison between typically developing children (TD) and CPNO. Out of 38 responses, the mean of TD is seen to be lower than the CPNO group in 6 responses. A further look at the data, shows that one response had the same mean for both groups (response no.3). Response no. 8 and 10 are related to taste/ smell sensitivity. The TD who have advantage of exposure to outside world on a larger magnitude, have more choices and preferences over the children with CP, who have restricted or limited exposure to outside world. Also, there is likelihood of oromotor dysfunction to be present in CP children. There may not be too much variation in the texture, taste or consistency in food for them On the other hand, the TD have tendency to be choosy 'always' or 'frequently'. This is in contrast to the findings by Ermer and Winnie Dunn that documented the profile of children without disability found a low incidence of behaviours in oral sensory sensitivity in them, when compared to children with autism and ADHD.In another study done by Jerome Anto Prakash and A. Vaishampayan, the mean of typically developing children was found to be higher than the mean of CP children.

In regard to response no. 17, 35 and 38, it is likely that TD also have some sensory issues which are overtly present and not reported by parents unless they cause a serious constraint in their day to day life. 


\section{International Journal of Science and Research (IJSR) \\ ISSN (Online): 2319-7064}

Index Copernicus Value (2013): 6.14 | Impact Factor (2015): 6.391

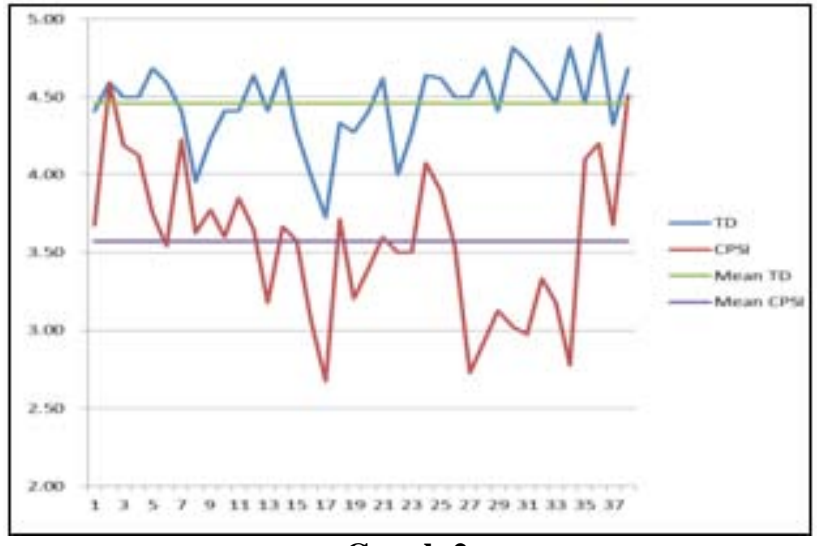

Graph 2

Graph 2 shows a comparison of the Typically Developed (TD) with the CPSI.

Although the t test is not significant for any response the means of all responses in TD group is higher than the mean of all responses in the CPSI group. Higher mean scores indicate desirable response and lower mean score indicates undesirable response. This proves that the CPSI group had more frequency of response to a given sensory stimulus which affected their performance. This is in agreement with the study published in Indian journal of OT in 2007, in which 40 out of 125 responses and 7 out of 14 components on caregiver's questionnaire showed a significant difference between typically developing children and children with Cerebral Palsy.

Graph 3 shows the comparison of typically developing children with PDD group. The t test is not significant for any of the 38 responses. However, as earlier the mean scores of PDD are lower than the mean scores of CPSI for all responses, which mean that PDD children have sensory issues predominantly affecting their functioning. Differences in sensory processing among people with autism have been well documented in the literature and the findings in this study add to the evidence. Sensory processing skills are fundamental to functional performance and therefore are likely to play a role in the variable developmental performance of people with PDD.

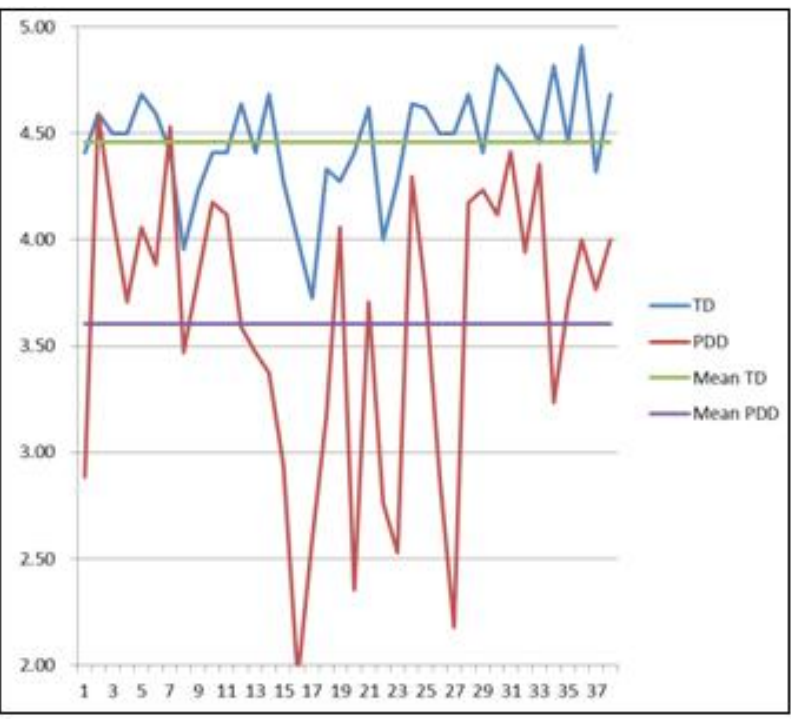

Graph 3

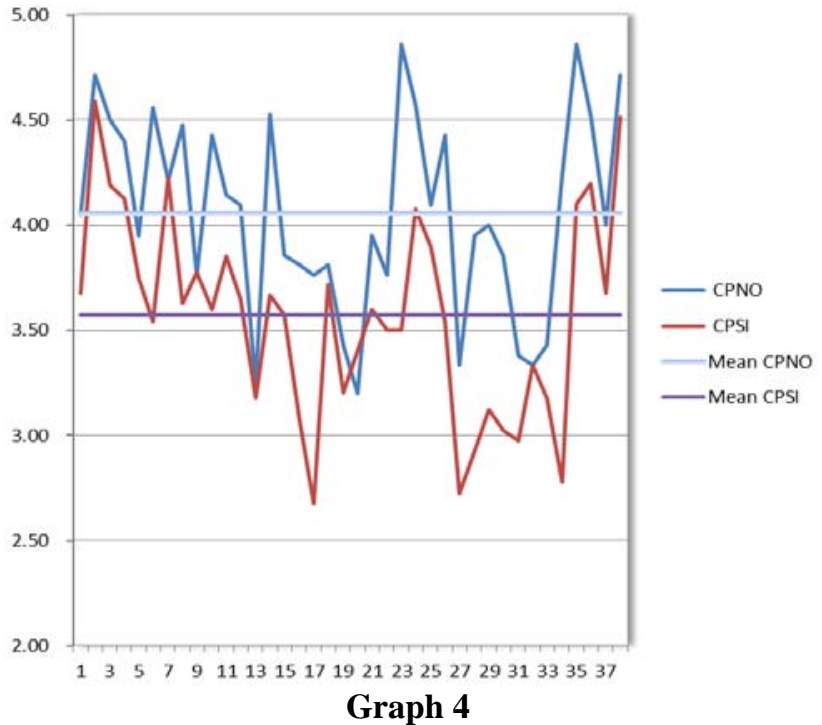

Graph 4shows the mean score of responses for each question by the caretaker of CPSI and CPNO.

The $\mathrm{t}$ test is not significant for any of the questions. However, if we look at the means of the two groups closely it is seen that 34 responses in the mean of CPNO group is higher than the mean of CPSI group. Response no. 32 has the same mean (3.33). The three means that are higher in CPSI group are response nos. 7, 9 and 20. These are from the sections of tactile sensitivity, taste/smell sensitivity and under-responsive /Seeks sensation respectively. It appears that these 3 are the responses where CPSI are behaving differently to CPNO group. The incidence of poor registration is observed in CP quadriplegic and ataxic than in athetoid and diplegic(3). The CPSI group had larger number of Quadriplegic (18) and ataxic (10) in comparison to CPNO (7 \& 1 respectively). Hence the lower mean in tactile sensitivity is understandable.

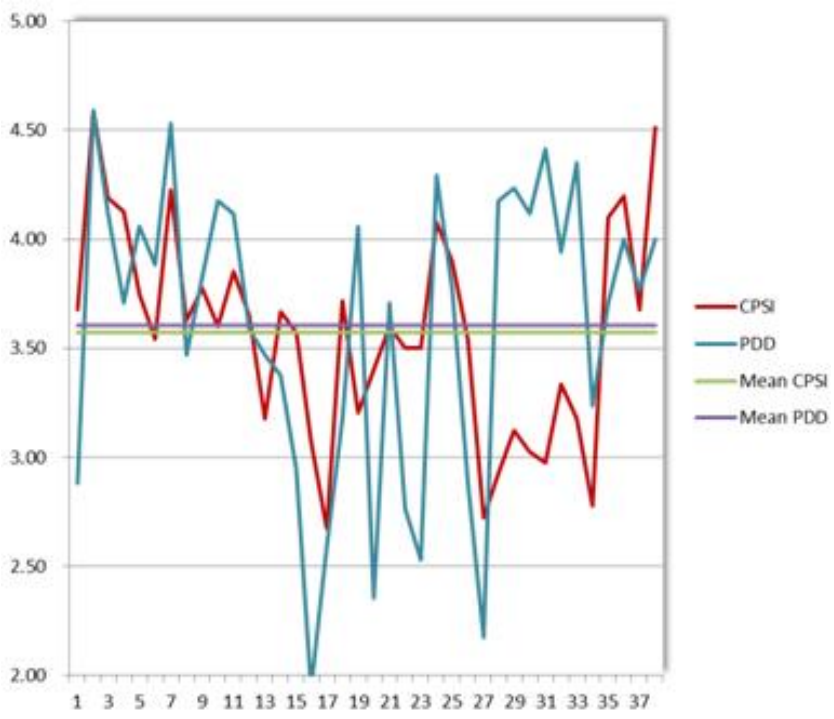

Graph 5

Graph 5 shows a comparison of mean responses for 38 questions covered in the SSP for CPSI group and PDD group.The t test for each response is not significant for any of the 38 questions. Out of 38 questions there are 19 questions for which the mean of CPSI group is higher 


\section{International Journal of Science and Research (IJSR) \\ ISSN (Online): 2319-7064 \\ Index Copernicus Value (2013): 6.14 | Impact Factor (2015): 6.391}

showing that CPSI group is seemingly better than PDD group. However, sections like tactile sensitivity, taste/smell sensitivity and low energy /weak are predominantly having smaller mean by CPSI group, indicating that these sections should be prioritized while checking SI issues in CP children.

\section{Conclusion}

There is a distinct difference in Cerebral Palsy children with sensory issues (CPSI) and children with pervasive developmental disorders (PDD).

The Short Sensory Profile has shown the trend of higher scores in typically developing children and CPNO then CPSI and PDD.

Although sensory processing problems have not explicitly been included in diagnostic criteria for children, some performance difficulties may be associated with poor sensory processing, specific to particular disability.

\section{Acknowledgement}

My sincere thanks to Dr. R. Sharma, Director of the Institute for allowing me to conduct this study. Thanks are due to Mrs. P.V. Rege for inspiring me on studying this subject on Sensory Integration. I would also like to acknowledge the support given to me by Mrs. S. Jayavant. Lastly, I express my gratitude to all the caretakers of the subjects who readily accepted to be the part of this study.

\section{References}

[1] R. Siebes,

L.Wijurocks,A.Vermeer (2007)Developmental Medicine and Child Neurology,Vol.44,issue 9, 593-603

[2] Scott D. Tomcheck, Winnie Dunn (2007).Sensory processing in children with and without autism: a comparative study using the short sensory profile. American Journal of Occupational Therapy 61,No.2, 190-200.

[3] A. Jerome Anto Prakash and A. Vaishampayan (2007) .A preliminary study on the sensory processing abilities of children with CP and typical children on the sensory profile. Indian Journal of Occupational Therapy Vol.39 no.2,27-34.

[4] S. M. Pedankatti (2005). A comparison of the performance of children with and without learning disability on the sensory profile tool. Indian Journal of Occupational Therapy Vol.36:No.3, 63-69.

[5] R.Watling, J. Deitz, O. White(2001).Comparison of Sensory profile scores of young children with and without autism spectrum disorders. American Journal of Occupational Therapy.Vol.55,No.2

[6] Bumin G, Kahiyan H (2001), Effectiveness of 2 different sensory integration programs for children with spastic diplegic cerebral palsy - Disability and Rehabilitation 23(9): 394-399

[7] Sensory Profile User's manual. Winnie Dunn .(1999)The Psychological Corporation.
[8] J.Ermer, W. Dunn(1998). The sensory Profile: A discriminant analysis of children with and without disabilities.American Journal of Occupational Therapy,Vol.52no.4,283-290

[9] Blanch E, Botticelli T. Hallway m (1995). Combining Neurodevelopmental treatment and sensory integrative principleas - An approach to pediatric therapy. San Antonio, Texas. Therapy Skill Builders

[10] Jane Case Smith and Jane Clifford O'Brien, Occupational Therapy for children, sixth edition. 